\title{
Architectural metabolic forms
}

\author{
I. W. Foged \\ Department of Architecture and Design, Aalborg University, Denmark
}

\begin{abstract}
Forms in nature are principally based on the ability to receive and distribute energy to maintain themselves. The purpose of the presented research is to take this basis as the fundamental constituents of creating forms and organisations in architecture, so to have metabolic capacities. Enabled by algorithmic digital models with integrated physical properties of the environment and the solid matter applied in the model, the work studies the formation processes and final forms of an architecture derived by energy transfer. Rather than thinking in terms of a priori defined geometries and typologies, formations and forms are the resultant of points distributed in space from ground upward with various densities effecting connections, enclosures and volumes. Performance evaluation and communication to the designer is provided directly in the model during formations and allow there through a more precise understanding of why a form is developing how it is. Concretely, the derived forms are evolved on the basis of a continuous feedback between solar energy, which spreads downward, the distributed mass that holds and spreads energy through air and the energy from the earth such as water and 'nutrients' travelling upward. The results illustrate the ability to organise forms that exhibit high performance in holding and allocating energy, such as thermal storage and spreading of heat. Furthermore, the communicative formations allow the designer to apply design parameters to parallel numerical optimisation with proportional aspect so fundamental to architectural expression. At its best, contemporary architecture simulate on forms to 'check' there performance. This work sets the conditions of context as the basis for development, so that forms only evolve if they have the energy to support it.

Keywords: metabolism, generative algorithms, architectural systems.
\end{abstract}




\section{Introduction}

\subsection{Inspiration from nature}

When architecture again looks to nature for inspiration, it is with common reference and platform in the extraordinary studies, experiments and built works of architect Antoni Gaudi moving far beyond his contemporaries through a mindset and development of exploratory models that continue to aspire as described by Burry [1] and Burry et al. [2]. An extension into several other studies was performed by architect and engineer Frei Otto and Rasch [3], who expanded the approach to minimal structures in various materials and methods. The merge of architectural considerations to tactility, proportions, elegance and engineering considerations of performative systems and structures brought novel results in works that are under admiration and construction today in Barcelona, through La Sagrada Familia, and Stuttgart, through Stuttgart Main Station.

What made their work move beyond anything else can be argued to be their ability to construct methods and operational systems they as designers could interact with, while the system inherently performed towards structural integrity. Due to the obvious excellence achieved, many contemporary experimental architects and researchers inhabit their approaches, to a degree of converting these physical models to digital models such as Pigram [4] and Killian [5].

\subsection{Related work}

The exact ability to apply computational power in digital models attempts not only to realise the projects, initiated by Gaudi and Otto, but also to search new domains that pursue the idea of constructing methods and models that move beyond conventional drafting techniques, whether it being sketching in hand or modelling in commercial software packages.

In line of inspiration from nature, architecture has taking a turn towards a both more concrete and abstract application of natures design systems. The former, concrete, through a biomimetic approach e.g. as systematic observations of how fibres are organised in plants and trees to achieve bending behaviour as investigated by Schneigger et al. [6], Reichert and Menges [7], Hensel [8] and how animals are proportioned and scaled through allometric measures, being the relation between size and shape as elaborated on by Niklas [9]. The latter, more abstract path, is less direct and based not on form and structure observations, but on descriptions of growth and development of forms through generative systems that iterate explicit procedures towards the formalisation and organisation of many elements, becoming one whole as seen in architecture agendas through complex organisations of many elements forming into emergent structures in the work of Sabin and Jones [10], Andrasek [11] and Oxman [12]. 


\subsection{Metabolic relations}

Organisation of elements (matter) that form into voids and structures are endless, and thus the intent and strategy for their organisation becomes more important. This work orientates its approach to metabolic relations, which is the relation between form, scale and energy as described by Weinstock [13]. In nature an example is found in the termite mound, which form is derived from several form-to-energy relations including the overall morphology to shade and ventilate the surface and to drag air through the structure via underground channels that is located and formed to increase cooling of air before it rises through the mound.

Of importance is to both convert and transfer energy to grow and maintain itself.

In architecture, architects have interest in forms for their expressive character and for their functional abilities. Scale or size is often a consequence of a specific task given and energy is of growing concern in a world undergoing climatic and social changes of power and the control of energy resources. All three aspects are related in the organisation of metabolic organisations and serve as ideological platform in the presented work.

\section{Computational approach}

An approach that lends not from the direct use of forms in nature, but from the conditions that is the basis for natural morphogenesis, is greatly appreciating the advances in computational sciences over the last five to six decades. In the seminal book 'A New Kind of Science', Steven Wolfram [14] sets a path for searching and solving complex phenomena based upon the properties and interactions between small units and exemplifies this with e.g. cellular automata governed by simple rule sets. Each simple rule set provides different advanced formations. Applying growth strategies for an environmental architecture with embedded logic of construction enables thus a bypass of the current agenda in sustainable architecture that at its best simulate buildings after their creation.

The logic of a digital building block with all information, analogous to a cell containing all information, about its physical properties and reactive patterns to external stimuli of environment and other building blocks allow descriptions of relative simple elements, but with regional and global advanced behaviour as seen in cellular automata.

A potential is, that we can inform about environmental properties and material properties, or collectively as properties of matter, and allow environmental architectural forms to evolve, both as a platform for studying the formations, to understand the relations between building blocks and environment, and as an engine for performative architectural organisations, beyond a priori determined design suggestions. 


\section{Metabolic architectural forms}

\subsection{Framework}

In nature, forms can only grow or persist as long as they have the energy to do so according to Weinstock [13]. A relation between the size and organisation of a form is thus in direct relation to the energy available. The organisation of a tree structure reaches equilibrium between the spreading of its branches and leaves to receive maximum energy from the sun, while being able to transport water and nutrients upward through the roots from the ground. A form is a direct derivative of the 'forces acting on it' described in the seminal work 'On Growth and Form' by Thompson [15] combined with the initial growth logics of the system. These logics are referred to as the genotype (algorithm), where the resultant form, the phenotype, is based upon the interaction of the environment and the genotype. The developed procedural method and content of the developed algorithm is described below. Environment is within the presented research based upon solar energy and energy from the growth base surface.

The algorithm and environment is written in Processing, a Java based language and interface developed by Casey Reas and Ben Fry [16]. The interface allows the designer to observe the formations as they develop, being an essential part of further developments and integration of the algorithm in architecture.

\subsection{Conditions and relations}

The graphs below illustrate relations and conditions inserted into the algorithm. Algorithms have been developed to illustrate plant growth, such as the development of L-Systems, by biologist Aristid Lindenmayer and Prusinkiewicz [17] and the dynamic exchange between plants and the environment, such as stomata patterns correlation with cellular automata patterns, discovered by Peak et al. [18]. However, as mentioned above, these algorithms are not oriented towards architectural production and thus need to be confronted with performance measurements related to architecture and engineering. Learnt from nature in this work, is the ability to grow where there is correlation between energies and then to move on to architectural evaluation criteria's of thermal storage to regulate temperature deviations, heat transfer to evaluate spatial temperature spreading, heat gain on surface to organise matter for absorption, colouring of materials and principles of building techniques that applies logics of how each building block (point within the generative models) can be lain on top of a previously created layer of blocks.

Figure 1. Decrease of seed energy as a function of distance to the base surface seed. 


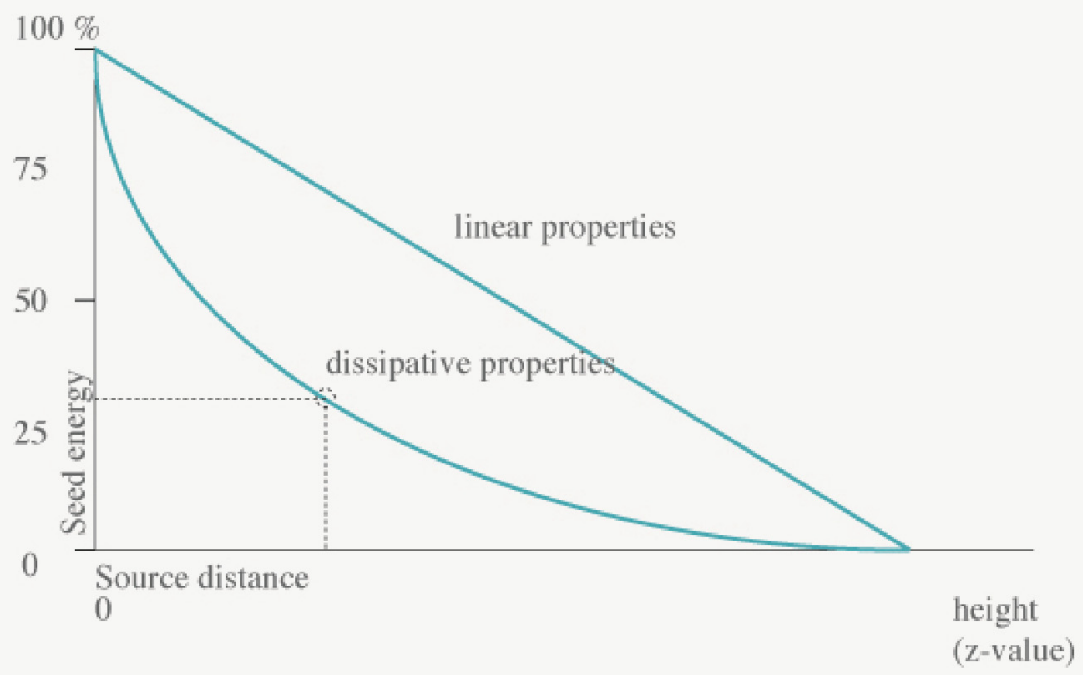

Figure 1: Seed energy.

Figure 2. Solar energy is calculated as function of the solar constant to the insolation factor.

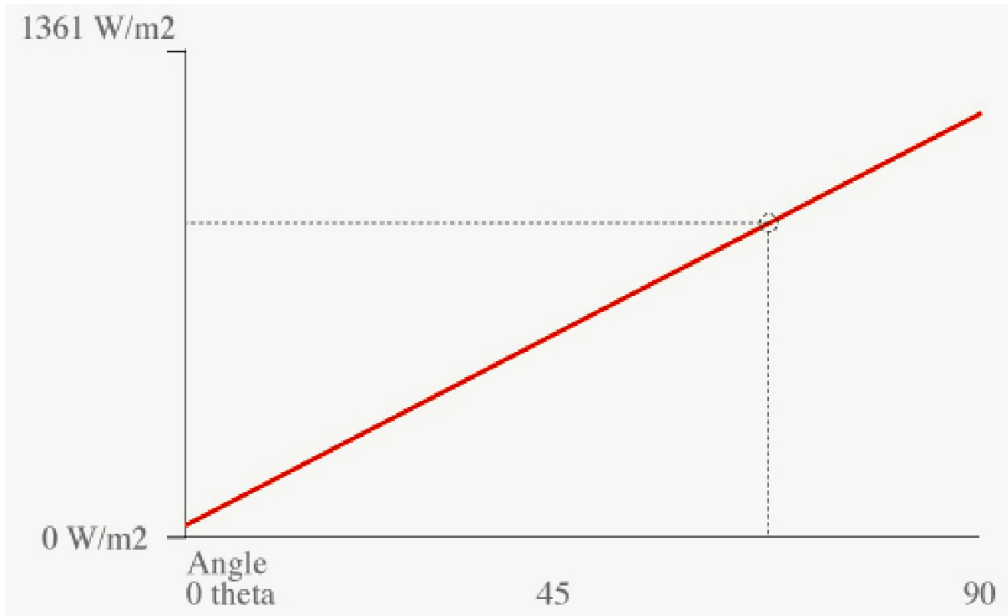

Figure 2: Solar energy.

Figure 3. Growth condition as a correlation between seed energy and solar energy requires that more than $25 \%$ of the maximum energy of both solar energy and seed energy is present. Above this threshold is the development initiated based upon the total energy of solar energy + seed energy. 


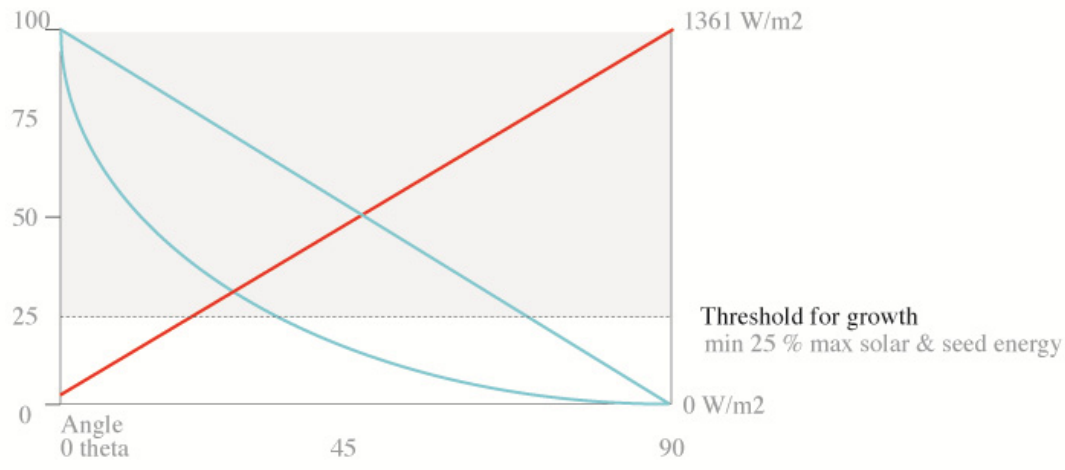

Figure 3: Conditions for initial and continuous growth.

Figure 4. The g-value is a coefficient factor from 0-1 that indicates the solar gain of the material. In months of low solar energy, we are interested in highenergy gains from the sun. The u-value is coefficient factor from $0-1$, indicating the heat transfer through a material. In months of low energy, we are interested in a low transfer to reduce heating demands.

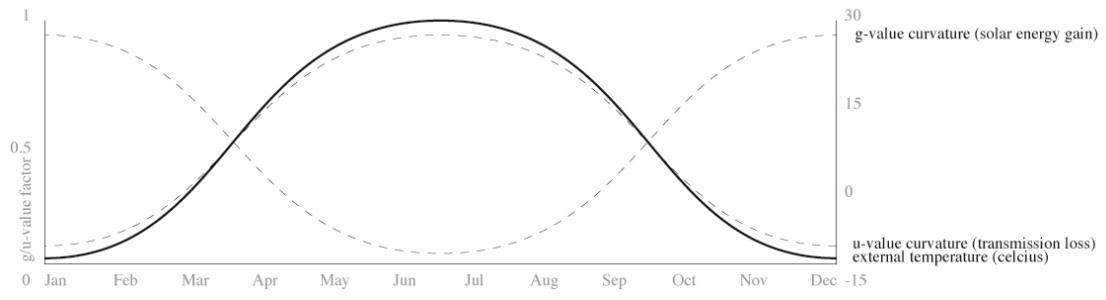

Figure 4: Relations between u-values, g-values and solar energy.

Figure 5. The graph illustrates the strategies for application of thermal storage (matter with high heat capacity) in relation to optimum distribution in relation to solar energy across the year.

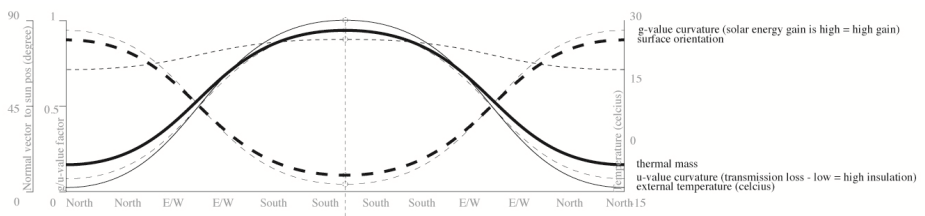

Figure 5: Relations for solar energy for distribution of matter for thermal storage. 


\subsection{Developed method}

The methodology inserts a generative algorithm that implements environmental conditions of energy from the sun in relation to energy on a base surface and energy on the developed forms. Base surface energy is understood as 'seed' energy representing positions of energy on the base surface and the intensity of these energies.

1) If the required correlation between the solar energy and seed energy are met conditions for growth are initiated and form development takes place. Initial seed position and energy intensity can be determined by the designer to specify site conditions or the algorithm can provide an initial seed organisation to start with. The latter is chosen for this paper, as the objective is to develop the method and algorithm and test its properties and performance, rather than a specific application. Additionally seed energy can be calculated for any distance from the initial seed following two logics; a) a linear decrease in strength or b) a dissipative decrease in strength based upon the distance from the initial base surface seed.

2) Forms develop based upon the idea of horizontal spreading from their centre (position of the seed) in all directions. The spreading, however, is asymmetrical based upon the inverse spreading of energy inward to the space framed by the curvilinear form. As in architecture, we are not merely interested in the maximum receiving of energy, but also its transfer into inner spatial organisations. The logic follows, the more energy received at any given point in the circular spreading from the centre of the seed, the longer from the centre it can travel.

3) A further distribution of matter is created in relation to organise thermal storage. This is inserted in the algorithm to balance the temperature deviations across time periods. For thermal mass to be organised in a meaningful architectural manner, optimum U-values (heat transfer coefficient) and optimum G-values (solar gain coefficient) are calculated at each point based upon the reception of energy at each point. The logic follows that the higher energy, the lower U-value (low heat transfer) and vice versa and the higher energy, the higher G-Value (low solar gain) and vice versa. The algorithm uses material thermal properties of concrete and water.

4) Based upon the distributed material properties, its volume and its orientation, a change in temperature is calculated at all points and the resultant stored energy in the form is distributed as heat inward towards the centre of the space, informing the designer of the performance of the algorithm as its develops form from the two initial conditions.

Figure 6. Seed is located at 1) within the surface grid. The sunray scans across the grid and follows below procedure. 2) Indicate the spreading of the matter from the seed. 3) Indicate the spreading of matter for thermal storage towards the seed. 4) Indicate the amount of heat movement inward.

Algorithmic procedure

1) Distribute seeds on surface

2) Pass over surface with sunrays

3) If intersection, calculate correlation of solar energy and seed energy 
4) If within growth domain, initiate growth based upon growth relations

5) Distribute first point set from seed location in all directions based upon energy

6) Calculate optimum U-values and optimum G-values

7) Distribute second point set from first set to create thermal storage

8) Calculate thermal energy stored

9) Calculate change of temperature

10) Calculate heat transfer from form towards centre of space.

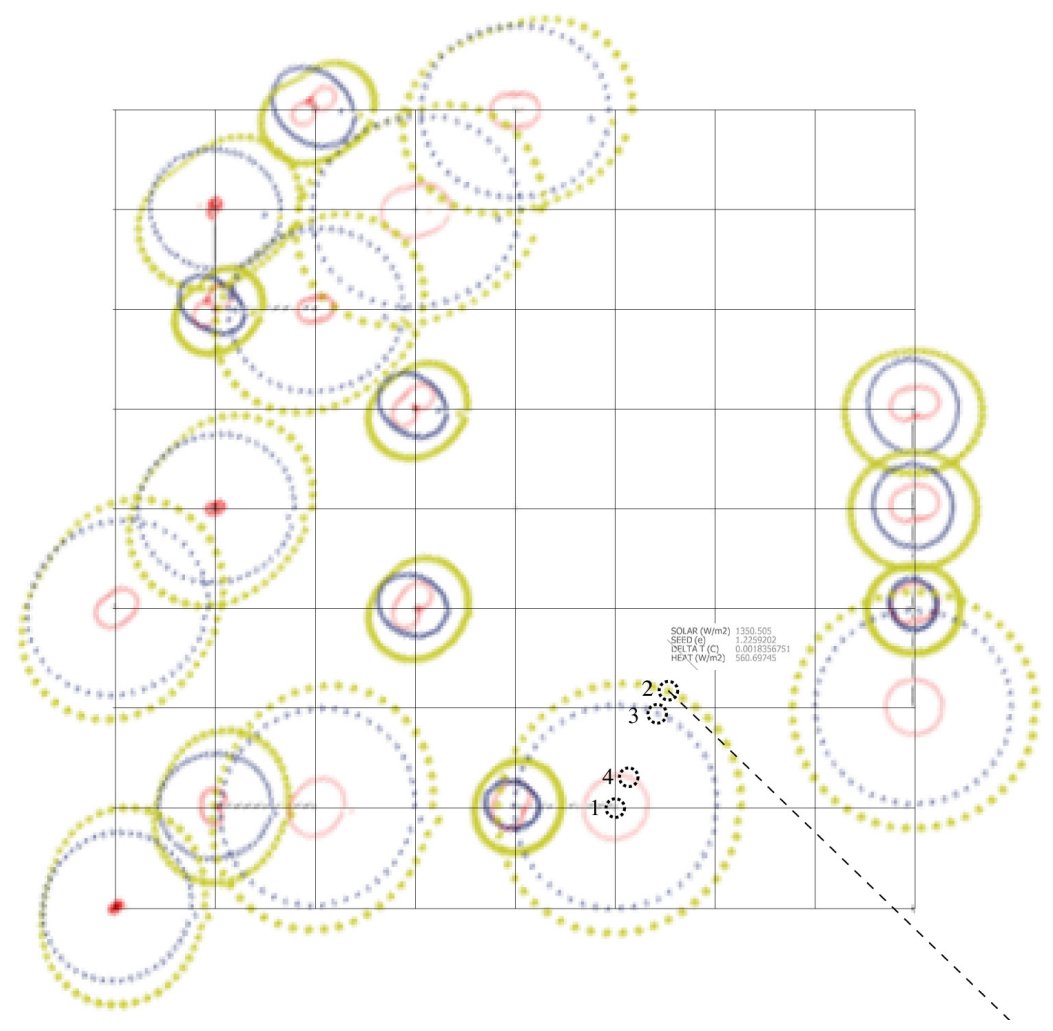

Figure 6: One layer distribution of matter (represented by points).

\subsection{Generative models and results}

For each run of the algorithm, a new formation takes place. This is not caused by altering properties of the algorithm, but altering environmental input and the correlation between these. The procedure of generating formations of 50000 elements has been run more than 50 times and indicates a consistent distribution of matter properties towards organisation of thermal storage and the derived distribution of heat energy. The model functions, during formation, to communicate input values of energies, the resultant form of these conditions and the resultant performance in terms of stored thermal energy, change of temperature and its radiation of heat energy inward. 


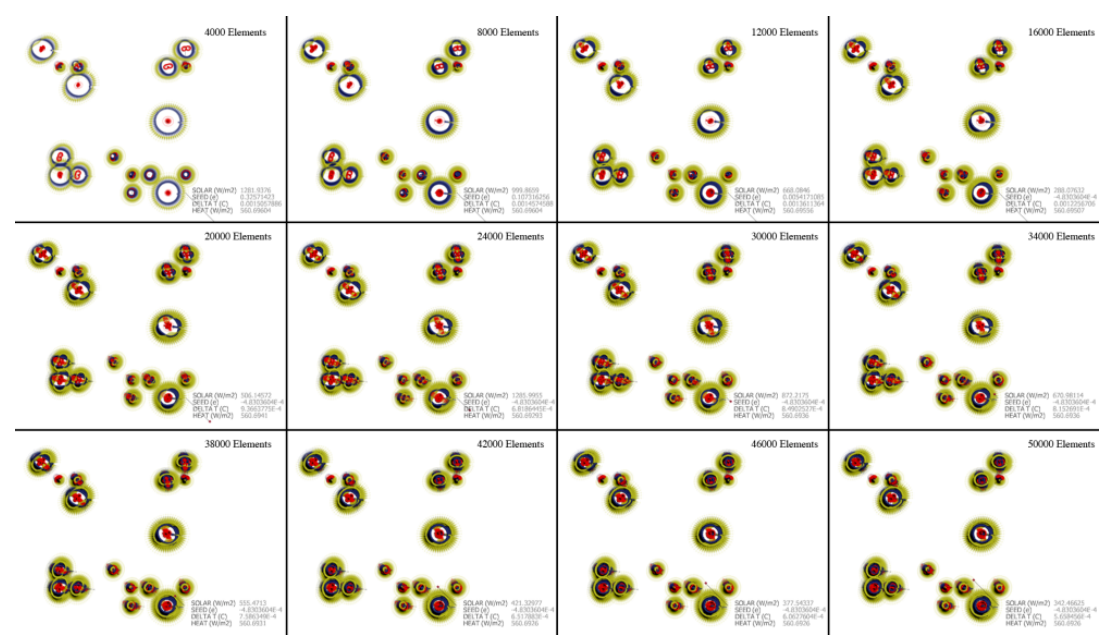

Figure 7: Matrix of formation in plan.

Figure 7. Matrix of formations in successive plan view for every 2000 elements distributed.

Figure 8. The formations illustrate the adapted outcome of the algorithm based on the relation to environment. If the sum of energies neglects to provide growth, the form is terminated in its further development.

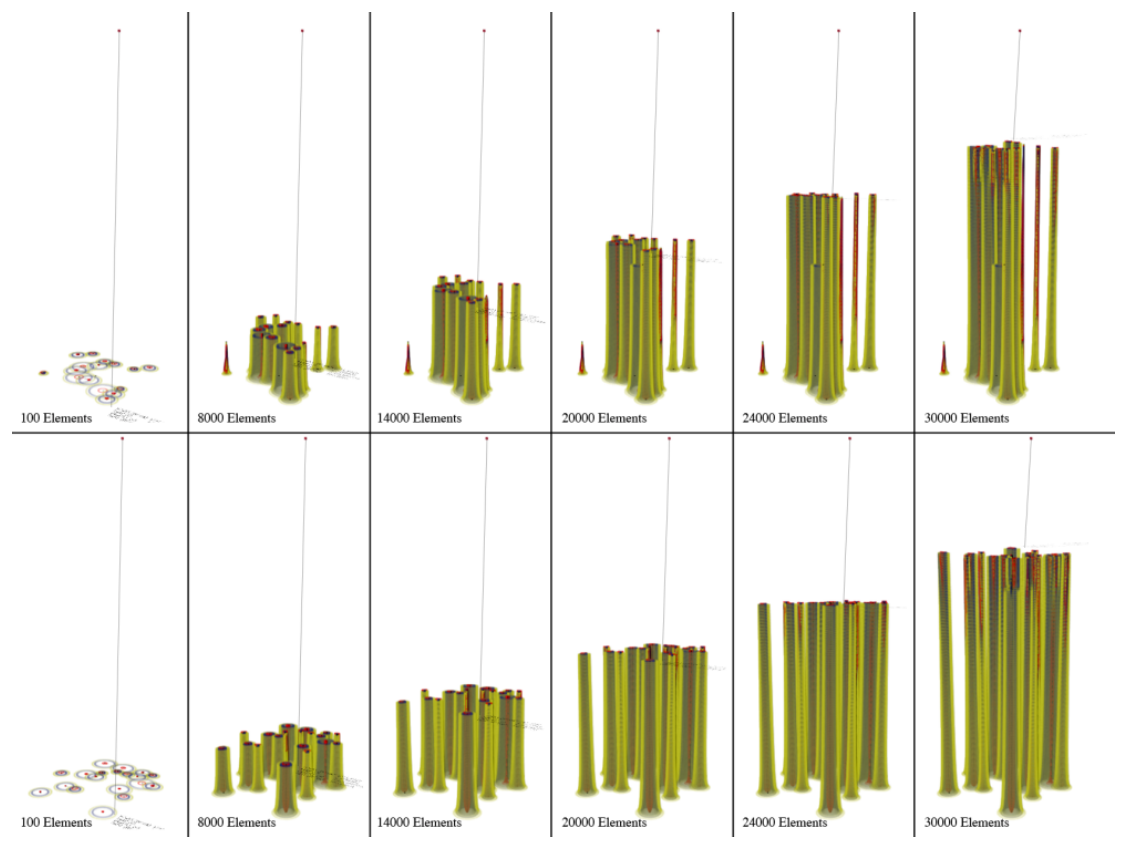

Figure 8: $\quad$ Two studies of formation of 20 forms. 


\section{Conclusions and future work}

The presented work learns from nature the process of constructing by the economy of energy, for then to apply architectural and engineering evaluation criterions to test the utility, usability and performance of the approach through a novel developed method and algorithm. The research identifies thereby the potential of a generic addition of matter to improve the absorption and transfer of energy in architecture and illustrates this through above examples.

This is found very suitable in testing its actual capacity for application in architecture. The work looks thus to a broader use than current focus to minimal structural designs, by instead suggesting a different approach towards studies and resultant maximum structures that also distribute matter in relation to aspects of energy use and human comfort zones.

By observing the representation of solid thermal matter and subsequent heat transfer in the models along the numerical data visible at every building block, the developed method and algorithm illustrate capacities of creating environmental architectural morphologies with lowered energy use and with expressive diversity and articulation in the final forms.

Ongoing distribution of matter in relation to context puts the local environmental conditions at the forefront of architectural creation and suggests great potentials for a further and broader application towards performative environmental and sustainable architectures.

Future development and application of the method and algorithm could include integration of more environmental factors, such as wind analysis, more defined contextual factors, such as neighbouring buildings and trees and organisation of internal spatial separations based upon the same logic of matter distribution in relation to energy available.

\section{References}

[1] Burry, M. Beyond Algorithm - Seeking Differentiated Structures Through Alternatives To Computational Design Processes. Pp. 334-347 MorphoEcologies (Ed. Hensel, M. and Menges, A.). AA Press London, 2006.

[2] Burry, M., Grifoll, J.C., Serrano, J.G. 2008. Sagrada Familia s. XXI Gaudi / now. Universitat Politecnica de Catalunya., 2008.

[3] Otto, F. and Rasch, B. Finding Form: Towards and Architecture of the Minimal. Axel Menges, 1996.

[4] Pigram, D. Studies in digital/physical models. http://supermanoeuvre.com/ pre-vault/

[5] Killian, A. Design Exploration through Bidirectional Modeling of Constraints. PhD Thesis. MIT. Boston, 2006.

[6] Schneigger, K, Rutzinger, S. Knippers, J. Scheible, F. Biomimetic Media Façade - Thematic Pavillion Expo 2012 Yeosu. International Adaptive Architecture Conference. London, 2011.

[7] Reichert, S., Menges, A. Adaptive Architecture Reflectivstruktur. Vordiplom. HfG Offenbach. 2006. 
[8] Hensel, M. Performance Oriented Architecture - Towards a Biological Paradigm for Architectural Design and the Built Environment. FORMakademisk vol. 3 Nr. 1. pp. 36-56, 2010.

[9] Niklas, K.J. Plant Allometry - The Scaling of Form and Process. The University of Chicago Press. London, 1990.

[10] Sabin, J., Jones, P.L. Nonlinear Systems Biology and Design - Surface Design. International Conference ACADIA Proceedings, 2008.

[11] Andrasek, A. Continuum - A Self-Engineering Creature-Culture. AD Wiley, 2006.

[12] Oxman, N. Material Based Design Computation, PhD Thesis, MIT, Boston, 2010.

[13] Weinstock, M. The Architecture of Emergence - The Evolution of Form in Nature and Civilisation, John Wiley and Sons Ltd., 2010.

[14] Wolfram, S. A New Kind of Science. Wolfram Media, 2002.

[15] Thompson, D.W. On Growth and Form. Dover Publications. New York, 1992.

[16] Reas, C., Fry, B. Processing. www.processing.org.

[17] Prusinkiewicz, P., Lindenmayer, A. The Algorithmic Beauty of Plants. Springer Verlag. New York, 1990.

[18] Peak, D., West, J.D., Messinger, S.M., Mott, K.A. Evidence for Complex, collective dynamics and Emergent, distributed computation in plants. PNAS. Vol. 101, no.4, pp. 918-922., 2004. 